\title{
TENSOR PRODUCTS OF COMMUTATIVE BANACH ALGEDRAS
}

\section{U.B. TEWAN, M. BUTA GN EMOLAA MADAN}

\author{
Department of Mathematics \\ Indian Institute of Technology \\ Kanpur-208016, U.P. \\ INDIA
}

ABSTRACT. Let $A_{1}, A_{2}$ be commutative semisimple Banach algebras and $A_{1} \theta_{\partial} A_{2}$ be their projective tensor product. We prove that, if $A_{1} \theta_{\partial} A_{2}$ is a group algebra (measure algebra) of a locally compact abelian group, then so are $A_{1}$ and $A_{2}$. As a consequence, we prove that, if $\mathrm{G}$ is a locally compact abelian group and $\mathrm{A}$ is a commutative semi-simple Banach algebra, then the Banach algebra $L^{1}(G, A)$ of A-valued Bochner integrable functions on $G$ is a group algebra if and only if $A$ is a group algebra. Furthermore, if $\mathrm{A}$ has the Radon-Nikodym property, then. the Banach algebra $M(G, A)$ of A-valued regular Borel measures of bounded variation on $G$ is a measure algebra only if $A$ is a measure algebra.

KEY WORDS AND PHRASES. Commutative semisimple Banach algebra, projective tensor product, group algebra, measure algebra, locally compact abelian group, RadonNikodym property.

AMS (MOS) SUBJECT CLASSIFICATION CODES (1970). Primary 43A10, 43A20, $46 J 35$.

1. INTRODUCTION.

Let $A$ be a commutative Banach algebra. We shall say that $A$ is a group algebra (measure algebra) if $A$ is isometrically isomorphic to $L^{1}(G)(M(G)$ ) for some locally compact abelian group G. Let $G$ be a locally compact abelian group and A be a commutative semi-simple Banach algebra. The space $L^{1}(G, A)$ of $A$-valued Bochner integrable functions on $G$ becomes a commutative Banach algebra (see [1], [2] and [3]). A natural question arises: when is $L^{1}(G, A)$ a group algebra? If $A=L^{1}(H)$ for some 
locally compact abelian group $\mathrm{H}$, then it is well known (Theorem 3.2 of [3]) that $L^{1}(G, A)$ is isometrically isomorphic to $L^{1}(G \times H)$. Thus $L^{1}(G, A)$ is a group algebra if $\mathrm{A}$ is a group algebra. We shall prove that the converse is also true. There is another way of looking at this problem. It is known that $\mathrm{L}^{1}(\mathrm{G}, \mathrm{A})$ is isometrically isomorphic to $\mathrm{L}^{1}(\mathrm{G}) \otimes_{\partial} \mathrm{A}$ (see 6.5 of [4]). Thus, if $\mathrm{A}_{1}$ and $\mathrm{A}_{2}$ are group algebras, then so is $A_{1} A_{2} A_{2}$ Conversely, we shall show that, if $A_{1}$ and $A_{2}$ are two commutative Banach algebras and $\mathrm{A}_{1} \otimes_{\partial} \mathrm{A}_{2}$ is a group algebra, then so are $\mathrm{A}_{1}$ and $A_{2}$. It seems proper to remark that we are concluding properties for $A_{1}$ and $A_{2}$, assuming corresponding properties for $\mathrm{A}_{1} \otimes_{\partial} \mathrm{A}_{2}$. This is in contrast to the approach of Gelbaum [5] and [6]. Our result for $L^{1}(G, A)$ readily follows from this. The main tool in our investigation is a theorem of Rieffel [7] characterizing group algebras. In this paper, Rieffel also characterized measure algebras. Accordingly, we investigate whether the fact that $A_{1} \otimes_{\partial} A_{2}$ is a measure algebra implies that $A_{1}$ and $\mathrm{A}_{2}$ are measure algebras. We shall show that this is indeed the case. As a consequence, we shall show that, if $\mathrm{A}$ is a commutative Banach algebra having the Radon Nikodym property and $\mathrm{M}(\mathrm{G}, \mathrm{A})$ is the Banach algebra of A-valued regular Borel measures of bounded variation on $G$, then $M(G, A)$ is a measure algebra only if $A$ is a measure algebra.

\section{PRELIMINARIES.}

Let $\mathrm{E}$ and $\mathrm{F}$ be Banach spaces. The projective tensor product of $\mathrm{E}$ and $\mathrm{F}$ (see [8]) is denoted by $E \otimes_{\partial} F$. Every element $t \in E \otimes_{\partial} F$ can be expressed as $t=\sum_{i=1}^{\infty} e_{i} \otimes f_{i}$, with each $e_{i} \in E$ and $f_{i} \in F$, such that $\sum_{i=1}^{\infty}\left\|e_{i}\right\|\left\|f_{i}\right\|<\infty$. The norm of $t$ is given by

$$
\|t\|_{\partial}=\inf \left\{\sum_{i}\left\|e_{i}\right\|\left\|f_{i}\right\|: t=\sum_{i} e_{i} \otimes f_{i}\right\}
$$

where the infimum is taken over all possible expressions of $t$.

Let $f \in E^{*}, g \in F^{*}$, and $t \in E \theta_{\partial} F$ with $t=\sum_{i=1}^{\infty} e_{i} \otimes f_{i}$. We define $f \cap t=\sum_{1} f^{\prime} e_{i} \cdot f_{i}$ and $g \circ t=\sum_{1}^{\infty} g\left(f_{i}\right) e_{i}$. The series defining $f \circ t$ and $g \circ t$ converge because $\sum_{1}^{\infty}\left\|e_{i}\right\|\left\|f_{i}\right\|<\infty$. It is obvious that $\| \mathrm{f}$ o $t\|\leq\| f\|\| t \|$ and $\|g \circ t\| \leq\|g\|\|t\|$. The norms here, as well as elsewhere, $\therefore$ fer to the norms 
in the spaces containing the elements. $t \rightarrow g \circ t$ and $t \rightarrow f o t$ define bounded linear maps from $E \otimes_{\partial} F$ to $E$ and $E \otimes_{\partial} F$ to $F$, respectively. These maps will be frequently used in the sequel.

Let $(S, \Sigma, \lambda)$ be a measure space and $X$ be a Banach space. $L^{1}(S, X)$ denotes the Banach space of $\mathrm{X}$-valued functions integrable with respect to $\lambda$. We shall often use the fact that $L^{1}(S) \otimes_{\partial} X$ is isometrically isomorphic to $L^{1}(S, X)$.

Gelbaum [5] and Tomiyama [9] have shown that, if A and B are commutative Banach algebras, then $\mathrm{A} \theta_{\partial} \mathrm{B}$ forms a commutative Banach algebra whose maximal ideal space is homeomorphic to the cartesian product of the maximal ideal spaces of A and $\mathrm{B}$. The maximal ideal space of a commutative Banach algebra A will be denoted by $\Delta(\mathrm{A})$. An element of $\Delta(\mathrm{A})$ will be regarded as a multiplicative linear functional (m.1.f.) of A. A11 the Banach algebras in our discussion will be taken to be commutative and semisimple. It is proved in [6] that $A \theta_{\partial} B$ has an identity if and only if both $\mathrm{A}$ and $\mathrm{B}$ have identities. It is also known [6] that $\mathrm{A} \otimes_{\partial} \mathrm{B}$ is Tauberian if $\mathrm{A}$ and $\mathrm{B}$ are Tauberian. The following lemma, though simple, does not seem to have appeared in print.

LEMMA 2.1. If $A \otimes_{\partial} B$ is Tauberian, then so are $A$ and $B$.

PROOF. Let us show that $B$ is Tauberian. It can be shown in the same way that A is Tauberian. Let $\mathrm{b} \in \mathrm{B}$ and $\epsilon>0$. Take $\phi \epsilon \Delta(\mathrm{A})$ and $\mathrm{a} \epsilon \mathrm{A}$ such that $\phi(\mathrm{a})=1$. Let $t=a \otimes b$. Choose $s \in A \otimes_{\partial} B$ such that $\hat{s}$ has compact support $K$ and $\|s-t\|<\epsilon$. Let $\mathrm{K}_{1}=\{\psi \in \Delta(\mathrm{B}):(\phi, \psi) \in \mathrm{K}\}$. Then $\mathrm{K}_{1}$ is compact. Let $\mathrm{x}=\phi \circ \mathrm{s}$. Then $\hat{\mathrm{x}}$ is supported in $\mathrm{K}_{1}$ and

$$
\|\mathrm{b}-\mathrm{x}\|=\|\phi \circ \mathrm{t}-\phi \circ \mathrm{s}\| \leq\|\mathrm{t}-\mathrm{s}\|<\epsilon .
$$

This proves that $\mathrm{B}$ is Tauberian.

Let $(S, \Sigma)$ be a measurable space and $X$ be a Banach space. Let $\mu$ be an $X$-valued set function on $\Sigma$. The total variation $V(\mu)$ of $\mu$ is defined for any $E \subset S$ as follows.

$$
V(\mu)(E)=\operatorname{Sup}\left\{\sum_{1}^{n}\left\|\mu\left(E_{i}\right)\right\|: E_{i}^{\prime} \text { s disjoint, } E_{i} \subset E \text { for } i \leq i \leq n\right\},
$$

the supremum being taken for all possible choices of $E_{i}$ 's. 
An $X$-valued measure on $(S, \Sigma)$ is a countably additive set function from $\Sigma$ into $X$. $\mu$ is said to be of bounded variation if $V(\mu)$ is finite. The space $\bar{M}(S, \Sigma, X)$ of $\mathrm{X}$-valued measures of bounded variation on $\mathrm{S}$ forms a Banach space under the norm $\|\mu\|_{V}=v(\mu)(S)$

Let $\lambda$ be a positive measure on $(S, \Sigma)$ and $L^{1}(S, X)$ be the Banach space of $X-$ valued functions on $S$, integrable with respect to $\lambda$. If $F \in L^{1}(S, X)$, then we can define the mapping $\mu_{F}: \quad \sum \rightarrow X$ by $\mu_{F}(E)=\int_{E} F d \lambda$. Then $\mu_{F}$ is an $X$-valued measure of bounded variation on $S$. Let $\mu \in \bar{M}(S, \Sigma, X)$. We say that $\mu$ has the derivative $F$ with respect to $\lambda$ if $\mu$ equals $\mu_{F}$ for $F \in L^{1}(S, X)$. We say that $X$ has the RadonNikodym property ( $\mathrm{X}$ has RNP) if every $\mathrm{X}$-valued measure $\mu$ of bounded variation on an arbitrary measurable space $(S, \Sigma)$ has a derivative with respect to $V(\mu)$. If $X$ is separable and the dual of a Banach space or is reflexive, then $X$ has RNP (see [10] and [11]). An example of a separable Banach space which does not have RNP is $L^{1}[0,1]$ (see $\left.[12]\right)$.

Let $\mathrm{G}$ be a locally compact abelian group and let $\mathrm{A}$ be a commutative Banach algebra. $M(G, A)$ denotes the Banach space of A-valued regular Borel measures of bounded variation on $G$. Suppose the range of every $\mu \in M(G, A)$ is separable. This is true if $A$ has RNP or if $G$ is second countable. Under these conditions, we can define the convolution of measures $\mu$ and $\nu$ belonging to $M(G, A)$. This makes $M(G, A)$ a commutative Banach algebra (see [13]). The algebra $L^{1}(G, A)$ is an ideal in $M(G, A)$ (see [14]). There is a natural isometric isomorphism from $M(G) \theta_{\partial} A$ into $M(G, A)$ (Theorem 4.2 of [15]). This is a Banach algebra isomorphism and, if A has RNP, then it is onto (Theorem 4.4 of [15]).

Let $\mathrm{A}$ be a commutative and semisimple Banach algebra and $\mathrm{m} \epsilon \Delta(\mathrm{A})$. Let $\mathrm{P}_{\mathrm{m}}=\{\mathrm{a} \in \mathrm{A}: \mathrm{m}(\mathrm{a})=\|\mathrm{m}\|\|\mathrm{a}\|\}$. Then $\mathrm{P}_{\mathrm{m}}$ is a cone in $\mathrm{A}$ and therefore introduces an order in $A$. Let $R_{m}=\left\{a-b: a, b \in P_{m}\right\}$. $m$ is said to be $L^{\prime}$-inducing if the following conditions are satisfied:

(1) $\quad\|m\|=1$

(2) $\mathrm{P}_{\mathrm{m}}$ is a lattice.

(3) If $a, b \in R_{m}$ and $a \wedge b=0$, then $\|a+b\|=\|a-b\|$. 
(4) If a $\in A$, then there exists unique elements, $a_{1}, a_{2} \in R_{m}$, such that $a=a_{1}+1 a_{2}$.

(5) Let $|a|=v\left\{\operatorname{Re}\left(e^{i \theta} a\right): \theta \in[0,2 \pi]\right\}$. Then $\|a||=|||a|\|$.

[V and $\wedge$ respectively denote supremum and infimum. $\operatorname{Re}(a)=a_{1}$ where $\left.a=a_{1}+1 a_{2}, a_{1} \in R_{m}\right]$. We note that if (1) - (3) hold, then $R_{m}$ forms a real abstract L-space in the sense of Kakutani [16], and hence $R_{m}$ is a boundedly complete lattice (see page 35 of [7]). Therefore, $|a|$ is well defined.

In [7], a L'-inducing m.1.f. is defined to be a m.1.f. which satisfies the following condition in addition to (1) - (5).

(6) For $a, b \in A,|a . b| \leq|a| \cdot|b|$.

However, White [17] has shown that a m.1.f. satisfying (1) - (5) automatically satisfies (6), and hence our definition is equivalent to that of [7]. We now state Rieffel's characterization of a group algebra.

THEOREM $R_{1}$. Let $A$ be a commutative semisimple Banach algebra. A is a group algebra if and only if

(a) every m.1.f. of $A$ is $L^{\prime}$-inducing, and

(b) A is Tauberian.

Let $A$ be a commutative semisimple Banach algebra and let $D$ be the collection of $L^{\prime}$-inducing m.1.f.'s of A. Consider the $\omega *$-topology on $D$. A continuous function $p$ on $D$ is said to be a D-Eberlein function if there exists a constant $k>0$ such that for any choice of points $m_{1}, \ldots, m_{n}$ of $D$ and scalars $\alpha_{1}, \ldots, \alpha_{n}$; we have

$$
\left|\sum_{1}^{\mathrm{n}} \alpha_{i} \mathrm{p}\left(\mathrm{m}_{i}\right)\right| \leq \mathrm{k}\left\|\sum_{1}^{\mathrm{n}} \alpha_{i} \mathrm{~m}_{i}\right\|_{A^{*}}
$$

The following theorem of Rieffel characterizes a measure algebra.

THEOREM $R_{2}$. Let A be a commutative Banach algebra and let $D$ be the set of $L^{\prime}-$ inducing m.1.f.'s of $A$. Then $A$ is a measure algebra if and only if

(i) D is a separating family of linear functionals of A,

(ii) D is locally compact in the $\omega *-t o p o l o g y$, and

(iii) every D-Eberlein function is the restriction to D of the Gelfand transform of some element of A. 
The 'if' part is nothing but Theorem B of [7]. The 'only if' part follows from the following and the familiar properties of Fourier-Stieltjes transforms. PROPOSITION 2.1. The L'-inducing m.1.f.'s of $M(G)$ are precisely those that are given by $\Gamma$, the dual of $G$.

PROOF. Let $S$ be the structure semigroup of $M(G)$ (see 4.3 of [18]). M(G) can be identified (3.2 of [18]) with a weak*-dense subalgebra of $M(S)$. Under this identification, the m.1.f.'s of $M(G)$ are given by $\hat{S}$, the collection of semicharacters of $\mathrm{S}$. Let $\mathrm{f} \in \hat{\mathrm{S}}$. Then, using the arguments of Proposition 2.5 of [7] (see also Proposition 2.8 of [7]), we can prove that $f$ represents an L'-inducing m.1.f. if and only if $|\mathrm{f}(\mathrm{s})|=1$ for all $\mathrm{s} \epsilon \mathrm{S}$. By 4.3.3 of [18], uf $\epsilon \hat{\mathrm{s}}:|\mathrm{f}|=1\}$ is the canonical image of $\Gamma$ in $S$. This proves our proposition.

\section{MAIN RESULT.}

Our main result is the following theorem. All other results are derived as a consequence of this.

THEOREM 3.1. Let $A_{1}$, $A_{2}$ be commutative semisimple Banach algebras and

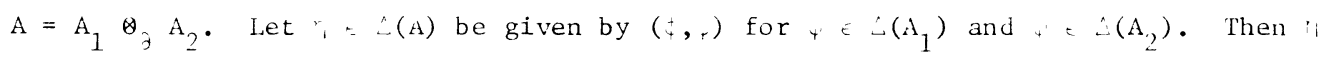
is $L^{\prime}$-indusing if and oniy if ; and are L'-inducing.

PRUOF. Suppose $n$ is L'-inducing. We shall show that i satisfies (1) - (5)

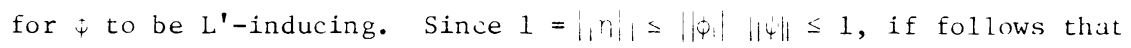
$i_{i} \phi\left\|_{1}=\right\|_{1}=1$. Let $P=\left\{t \in A: n(t)=\| t_{i}\right\}$ and $P_{\phi}=\left\{r \in A_{1}: \phi(r)=\| r r\right.$ Choose a fixed $s \in A_{2}$ such that $\psi(s)=\|s\|=1$. Let $t \in P_{\eta}$ and $r={ }_{4} o t$. Then $p(r)=\psi(\psi \circ t)=n(t)=|i t|=|| r_{i}$. Therefore, $r \in P_{\phi}$. On the other hand, if $r=P_{\phi}$, then $\eta(r \& s)=\phi(r)_{\psi}(s)=\phi(r)=\left\|r_{i}=\right\| r\|\|_{i} s_{i}=s_{i 1}$ and so $r \Leftrightarrow s \in P_{\| i}$. Thus we have shown that, if $t_{1}, t_{2} \in A$ and $t_{1} \geq t_{2}$, then $40 t_{1} \geq 40 t_{2}$, and, if $r_{1}, r_{2} \in A_{1}$ and $r_{1} \geq r_{2}$, then $r_{1} \otimes s \geq r_{2} \otimes s$.

Now, let $r_{1}, r_{2}=P_{0}$. Then it is easy to see that $r_{1} \vee r_{2}=$ $r o\left(\left(r_{1} \otimes s\right) \vee\left(r_{2} \otimes s\right)\right)$ and $r_{1} \wedge r_{2}=t o\left(\left(r_{1} \otimes s\right) \wedge\left(r_{2} \otimes s\right)\right)$. For example, if $r=r o\left(\left(r_{1} \otimes s\right) \vee\left(r_{2} \otimes s\right)\right)$, then, since $\left(r_{1} \otimes s\right) \vee\left(r_{2} \otimes s\right) \cdot r_{1} \otimes s$, ii follows that $r \geq r_{1}$. Similarly, $r \geq r_{2}$. On the other hand, if $r^{\prime}>r_{1}$ and $r^{\prime}$, $r_{2}$, then $r^{\prime} \otimes s \geq r_{1} \otimes s$ and $r^{\prime} \otimes s \geq r_{2} \otimes s$. Therefore, $r^{\prime} \cdot r$. Note that $r_{1} v^{\prime} r_{2}$, ind 
$r_{1} \wedge r_{2}$ depend only on $r_{1}$ and $r_{2}$ and not on $s$. Therefore, $P_{\phi}$ is a lattice. We can also see that $\left(r_{1} \vee r_{2}\right) \otimes s=\left(r_{1} \otimes s\right) \vee\left(r_{2} \otimes s\right)$ and $\left(r_{1} \wedge r_{2}\right) \otimes s=\left(r_{1} \otimes s\right)$ $\left(r_{2} \otimes s\right)$. For example, it is obvious that $\left(r_{1} \vee r_{2}\right) \otimes s \geq\left(r_{1} \otimes s\right) \vee\left(r_{2} \otimes s\right)$ and furthermore,

$$
\begin{aligned}
\|\left(r_{1} \vee r_{2}\right) & \otimes s-\left(r_{1} \otimes s\right) \vee\left(r_{2} \otimes s\right) \| \\
& =\eta\left[\left(r_{1} \vee r_{2}\right) \otimes s-\left(r_{1} \otimes s\right) \vee\left(r_{2} \otimes s\right)\right] \\
& =\phi\left[r_{1} \vee r_{2}-\psi \circ\left(\left(r_{1} \otimes s\right) \vee\left(r_{2} \otimes s\right)\right)\right]=0 .
\end{aligned}
$$

Next, if $t \in R_{\eta_{1}}$ and $r \in R_{\phi}$, then $\psi \circ t \in R_{\phi}$ and $r \otimes s \in R_{n}$. Moreover, all the above relations are true for $r_{1} \vee r_{2}$ and $r_{1} \wedge r_{2}$ for $r_{1}, r_{2} \in R_{\phi}$. Now, let $r_{1}, r_{2} \in R_{\phi}$ and $r_{1} \wedge r_{2}=0$. Then $r_{1} \otimes s, r_{2} \otimes s \in R_{\eta}$ and $\left(r_{1} \otimes s\right) \wedge\left(r_{2} \otimes s\right)=0$. Therefore, $\left\|r_{1} \otimes s+r_{2} \otimes s\right\|=\left\|r_{1} \otimes s-r_{2} \otimes s\right\|$, and hence $\left\|r_{1}+r_{2}\right\|=\left\|r_{1}-r_{2}\right\|$. Hence $\phi$ satisfies (3).

Suppose now that $r \in A_{1}$. Then $r \otimes s \in A$ and $r \otimes s=t_{1}+i t_{2}$, with $t_{1} t_{2} \in R_{n}$. Then $r=\psi o(r \otimes s)=\psi \circ t_{1}+i \psi \circ t_{2}$. Also, if $r=r_{1}+i r_{2}=r_{3}+i r_{4}$ for $r_{i} \in R_{\phi}$, then $r \otimes s=r_{1} \otimes s+i r_{2} \otimes s=r_{3} \otimes s+i r_{4} \otimes s$. Therefore, $r_{1} \circ s=r_{3} \circ s$ and $r_{2} \otimes s=r_{4} \otimes s$. Hence, $r_{1}=r_{3}$ and $r_{2}=r_{4}$. We have also shown that $(\operatorname{Re} r) \otimes s=\operatorname{Re}(r \otimes s)$. Thus $\phi$ satisfies (4). We now show that $\phi$ satisfies (5). Let $r \in A_{1}$. First, we show that $|r|=\psi \circ|r \otimes s|$ and $|r \otimes s|=|r| \otimes s$. We have

$$
\begin{aligned}
\psi \circ|r \otimes s|-\operatorname{Re}\left(e^{i \theta} r\right) & =\psi \circ|r \otimes s|-\psi \circ\left(\operatorname{Re}\left(e^{i \theta} r \otimes s\right)\right) \\
& =\psi \circ\left[|r \otimes s|-\operatorname{Re}\left(e^{i \theta} r \otimes s\right)\right],
\end{aligned}
$$

for every $\theta \in[0,2 \pi]$. Therefore, $\psi \circ|r \otimes s| \geq|r|$. On the other hand, $|r| \geq \operatorname{Re}\left(e^{i \theta} r\right)$. Hence $|r| \otimes s \geq \operatorname{Re}\left(e^{i \theta} r\right) \otimes s=\operatorname{Re}\left(e^{i \theta}(r \otimes s)\right)$. Therefore, $|r| \otimes s \geq|r \otimes s|$, so that $|r| \geq \psi \circ|r \otimes s|$. Thus we have $|r|=\psi \circ|r \otimes s|$. Also, since $|r| \otimes s \geq|r \otimes s|$, we get

$$
\begin{aligned}
\||r| \otimes s-|r \otimes s|\| & =\eta[|r| \otimes s-|r \otimes s|] \\
& =\phi[|r|-\psi \circ|r \otimes s|]=0 .
\end{aligned}
$$

Therefore, $|r| \otimes s=|r \otimes s|$. Now $\|r\|=\|r\|\|s\|=\|r \otimes s\|=\||r \otimes s|\|=\||r| \otimes s\|=$ $\||r|\|\|s\|=\||r|\|$. This proves that $\phi$ satisfies (5). Hence $\phi$ is $L^{\prime}-i n d u c i n g$. We can show similarly that $\psi$ is $L^{\prime}$-inducing. 
Conversely, suppose $\phi$ and $\psi$ are $L^{\prime}$-inducing. We shall show that $\eta_{\text {is }} L^{\prime}$-inducing. It is obvious that $\|n\|=1$. Since $\phi$ is $L^{\prime}$-inducing m.1.f. of $A_{1}$, by Proposition 2.3 of [7], there exists a locally compact Hausdorff space $X$ and a positive regular Borel measure $\mu$ on $X$ such that $A_{1}$ is isometrically linear isomorphic and, under the order induced by $\phi$, order isomorphic to $L^{1}(X, \mu)$. The dual of $A_{1}$ is then represented by $L^{\infty}(X, \mu)$ and, under this representation, $\phi$ is represented by the constant function $\|\phi\|=1$ on $X$. Now, $A_{1} \theta_{\partial} A_{2} \simeq L^{1}(X, \mu) \theta_{\partial} A_{2} \simeq L^{1}\left(X, \mu, A_{2}\right)$. Hereafter, we shall not distinguish between elements of $A$ and $L^{1}\left(X, \mu, A_{2}\right)$ and, for $F \in L^{1}\left(X, \mu, A_{2}\right)$, statements like " $F \in A$ " will be used without explanation. For $F \in A$, we observe that $F \in P_{\eta}$ if and only if $\phi \circ F \in P_{\psi^{*}}$. This $18 s 0$, because $\|F\| \geq\|\phi \circ F\| \geq|\psi(\phi \circ F)|$. We also have

$$
\begin{aligned}
\|\mathbf{F}\|=\int\|\mathbf{F}(\mathbf{x})\| \mathrm{d} \mu(\mathbf{x}) & \geq\left|\psi\left[\int \mathbf{F}(\mathbf{x}) \mathrm{d} \mu(\mathbf{x})\right]\right| \\
& =\left|\int \psi(\mathbf{F}(\mathbf{x})) \mathrm{d} \mu(\mathbf{x})\right| .
\end{aligned}
$$

This shows that $F \in P_{\eta}$ if and only if $F(x) \in P_{\psi}$ a.e. ( $\mu$ ). Let $F_{1}, F_{2} \in P_{\eta}$. Using the continuity and other properties of the lattice operations, it is easy to prove that the function $F_{1} \vee F_{2}$ defined a.e. $(\mu)$ by $\left(F_{1} \vee F_{2}\right)(x)=F_{1}(x) \vee F_{2}(x)$, belongs to $L^{1}\left(X, \mu, A_{2}\right)$ and consequently defines an element of $P_{\eta}$. This proves that $P_{n}$ is a lattice. Other details involved in showing that $\eta$ is $L^{\prime}$-inducing are also now easy to verify and hence we omit them. This completes the proof of our Theorem.

Having proved our main theorem, we now proceed to give its consequences.

THEOREM 3.2. Let $A_{1}$ and $A_{2}$ be commutative semisimple Banach algebras. Then $A_{1} \theta_{\partial} A_{2}$ is a group algebra if and only if $A_{1}$ and $A_{2}$ are group algebras.

PROOF. As mentioned in the introduction, it is well known that, if $A_{1}$ and $A_{2}$ are group algebras, then so is $A_{1} \theta_{\partial} A_{2}$. The converse follows from Lemma 2.1, Theorem $R_{1}$ and Theorem 3.1.

The following is an immediate consequence of Theorem 3.2.

THEOREM 3.3. Let $\mathrm{G}$ be a locally compact abelian group and let $\mathrm{A}$ be a commutative semisimple Banach algebra. Then $L^{1}(G, A)$ is a group algebra iff $A$ is a group algebra. 
PROOF. The result follows from Theorem 3.2 and the fact that the Banach algebras $L^{1}(G, A)$ and $L^{1}(G) \theta_{\partial}$ A are isometrically isomorphic.

THEOREM 3.4. Let $A_{1}$ and $A_{2}$ be commutative semisimple Banach algebras and $A=A_{1} B_{2} A_{2}$. If $A$ is a measure algebra, then $A_{1}$ and $A_{2}$ are measure algebras.

PROOF. Let $D, D_{1}, D_{2}$ be the set of L'-inducing m.l.f.'s of $A, A_{1}$, and $A_{2}$ respectively. Theorem 3.1 implies that $D=D_{1} \times D_{2} \cdot$ Since $D$ satisfies condition (i) of Theorem $R_{2}$, it easily follows that $D_{1}$ and $D_{2}$ also satisfy this condition. Since $D$ is locally compact in the $\omega *-$ topology, $D_{1}$ and $D_{2}$ are also locally compact in the $\omega^{*}$-topology. It remains to show that $A_{1}$ and $A_{2}$ satisfy condition (iii) of Theorem $R_{2}$. We shall do this for $A_{2}$, the case of $A_{1}$ being similar. Since $A$ is a measure algebra, it has an identity. It follows that $A_{1}$ and $A_{2}$ have identities. Let $e$ be the identity of $A_{1}$. Let $p$ be a $D_{2}$-Eberlein function. Define the function $\mathrm{P}$ on $\mathrm{D}$ by $\mathrm{P}(\phi, \psi)=\mathrm{P}(\psi)$. Obviously, $\mathrm{P}$ is continuous. Moreover,

$$
\left|\sum_{1}^{n} \alpha_{i} P\left(\phi_{i}, \psi_{i}\right)\right|=\left|\sum_{1}^{n} \alpha_{i} P\left(\psi_{i}\right)\right| \leq k\left\|\sum_{1}^{n} \alpha_{i} \psi_{i}\right\| A_{2}^{*} .
$$

However, for any a $\in \mathrm{A}_{2}$,

$$
\begin{aligned}
\left\langle a, \sum_{1}^{n} \alpha_{i} \psi_{i}\right\rangle & =\left\langle e \theta a, \sum_{1}^{n} \alpha_{i}\left(\phi_{i}, \psi_{i}\right)\right\rangle \\
& \leq\left\|\sum_{1}^{n} \alpha_{i}\left(\phi_{i}, \psi_{i}\right)\right\|_{A^{*}}\|a\| .
\end{aligned}
$$

Therefore, $\left\|\sum_{1}^{n} \alpha_{i} \psi_{i}\right\|_{A_{2}^{*}} \leq \mid \sum_{1}^{R} \alpha_{i}\left(\phi_{i}, \psi_{1}\right) \|_{A^{*}}$. This shows that $P$ is a D-Eberlein function and therefore there exists $t \in A$ such that $\hat{t}(n)=P(\eta)$ for every $t \in \Delta(A)$. Choose $\phi \in \Delta\left(A_{1}\right)$ and let $b=\phi o t$. Then $\hat{b}(\psi)=p(\psi)$ for every $\psi \epsilon \Delta\left(A_{2}\right)$. This shows that $A_{2}$ satisfies condition (iii) of Theorem $R_{2}$ and the proof of our theorem is complete.

THEOREM 3.5. Let $\mathrm{G}$ be a locally compact abelian group and $\mathrm{A}$ be a commutative semisimple Banach algebra having RNP. Then $M(G, A)$ is a measure algebra only if $A$ is a measure algebra.

PROOF. The theorem follows from Theorem 3.4 and the fact that the algebras $M(G) \theta_{\partial} A$ and $M(G, A)$ are isometrically isomorphic under the hypothesis of our theorem. 


\section{REFERENCES}

1. HAUSNER, A. Group algebras of vector-valued functions, Bu11. Amer. Math. Soc. 62 (1956), 383 (abstract 493).

2. HAUSNER, A. The Tauberian theorem for group algebras of vector-valued functions, Pacific J. Math. 7 (1957), 1603-1610.

3. JOHNSON, G.P. Spaces of functions with values in a Banach algebra, Trans. Amer. Math. Soc. 92 (1959), 411-429.

4. SCHAEFER, H.H. Topological vector spaces, McMillan, New York, 1966.

5. GELBAUM, B.R. Tensor products of Banach algebras, Canad. J. of Math. $\underline{11}$ (1959), 297-310.

6. GELBAUM, B.R. Tensor products and related questions, Trans. Amer. Math. Soc. 103 (1962), 525-548.

7. RIEFFEL, M. A characterization of commutative group algebras and measure algebras, Trans. Amer. Math. Soc. 116 (1965), 32-64.

8. GROTHENDIECK, A. Products tensoriels topologiques et espaces nucleaires, Mem. Amer. Math. Soc. 16 (1955).

9. TOMIYAMA, J. Tensor products of commutative Banach algebras, Tohuku Math. J. $12(1960), 147-154$.

10: DUNFORD, N. and PETTIS, B. Linear operations on summable functions, Trans. Amer. Math. Soc. 47 (1940), 323-392.

11. PHILLIPS, R.S. On weakly compact subsets of a Banach space, Amer. J. of Math. 65 (1943), 108-136.

12. UHL, J.J. Jr. The range of a vector-valued measure, Proc. Amer. Math. Soc. 23 (1969), 158-163.

13. HUNNEYCUTT, J.E. Jr. Products and convolutions of vector-valued set functions, Studia Mathematica 41 (1972), 101-129.

14. TEWARI, U.B., DUTTA, M. and VAIDYA, D.P. Multipliers of group algebras of vector-valued functions, Proc. Amer. Math. Soc. 81 (1981), 223-229.

15. LAMADRID, J.G. Measures and Tensors, Trans. Amer. Math. Soc. 114 (1965), 98-121.

16. KAKUTANI, S. Concrete representations of abstract (L)-spaces and the mean ergodic theorem, Annals of Math. (2) 42 (1941), 523-537.

17. WHITE, A.J. Ordered Banach algebras, Journal London Math. Soc. Ser. 2 , 11 (1975), 175-178.

18. TAYLOR, J.L. Measure Algebras, Regional Conference Series in Math., no. 16, Conference Board of Mathematical Sciences, 1973. 


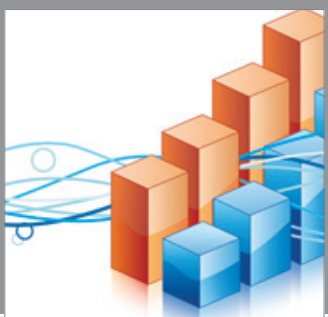

Advances in

Operations Research

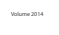

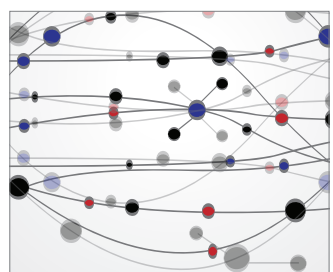

\section{The Scientific} World Journal
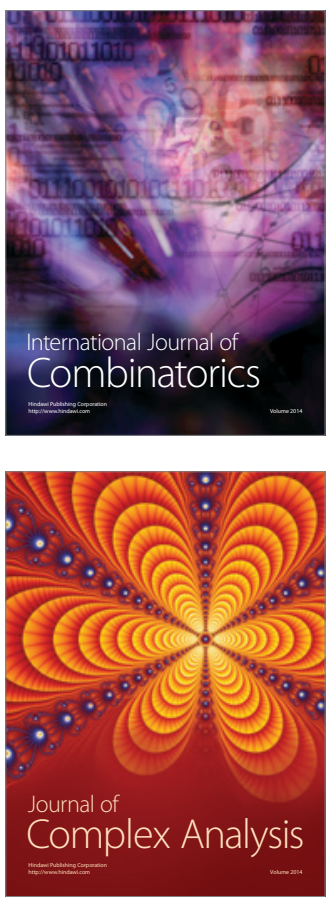

International Journal of

Mathematics and

Mathematical

Sciences
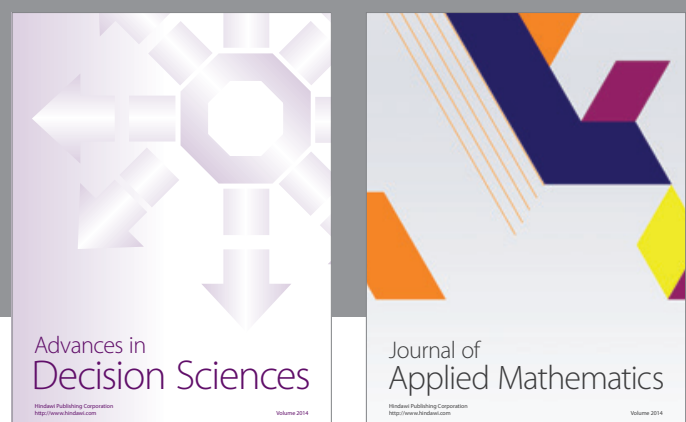

Journal of

Applied Mathematics
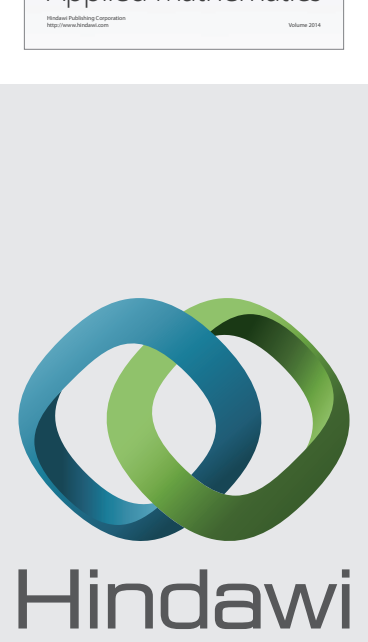

Submit your manuscripts at http://www.hindawi.com
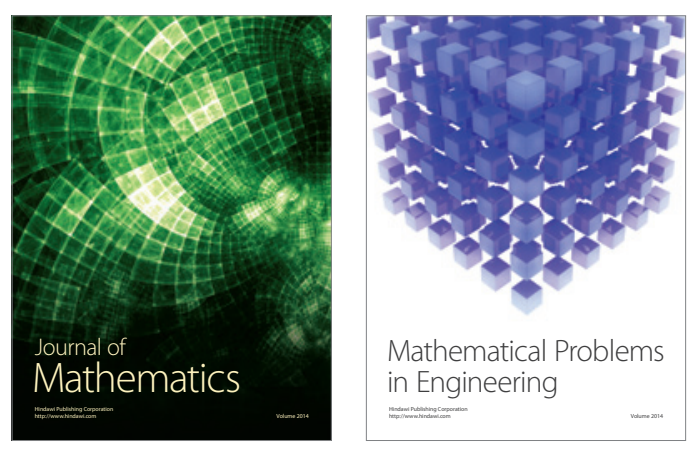

Mathematical Problems in Engineering
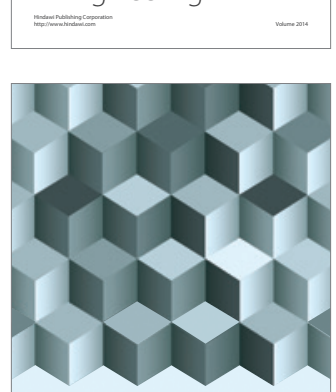

Journal of

Function Spaces
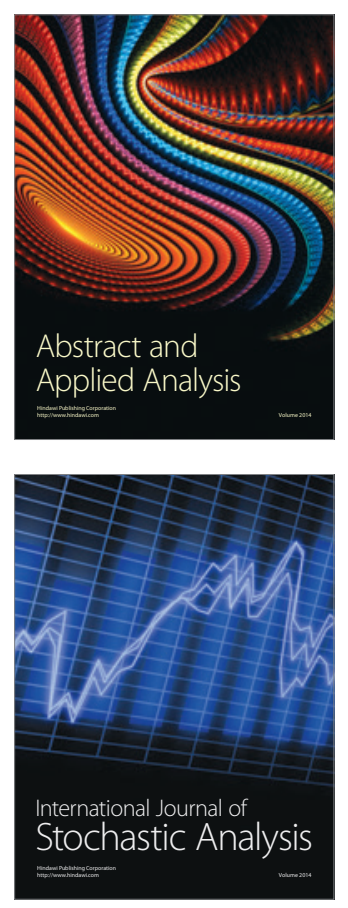

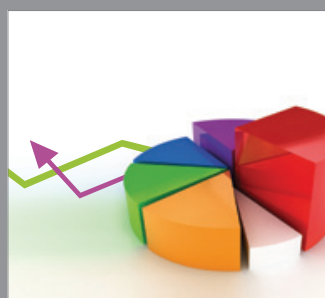

ournal of

Probability and Statistics

Promensencen
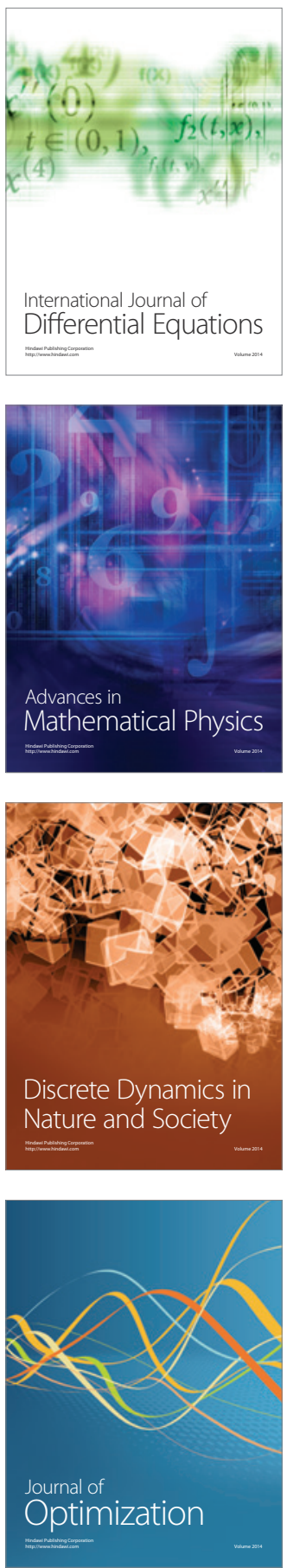